\title{
An investigation of back stress formulations under cyclic loading
}

\author{
El-Naaman, S. A.; Nielsen, K. L.; Niordson, C. F.
}

Published in:

Mechanics of Materials

Link to article, DOI:

10.1016/j.mechmat.2019.01.005

Publication date:

2019

Document Version

Peer reviewed version

Link back to DTU Orbit

Citation (APA):

El-Naaman, S. A., Nielsen, K. L., \& Niordson, C. F. (2019). An investigation of back stress formulations under cyclic loading. Mechanics of Materials, 130, 76-87. https://doi.org/10.1016/j.mechmat.2019.01.005

\section{General rights}

Copyright and moral rights for the publications made accessible in the public portal are retained by the authors and/or other copyright owners and it is a condition of accessing publications that users recognise and abide by the legal requirements associated with these rights.

- Users may download and print one copy of any publication from the public portal for the purpose of private study or research.

- You may not further distribute the material or use it for any profit-making activity or commercial gain

- You may freely distribute the URL identifying the publication in the public portal

If you believe that this document breaches copyright please contact us providing details, and we will remove access to the work immediately and investigate your claim. 


\title{
An investigation of back stress formulations under cyclic loading
}

\author{
S.A. El-Naaman, K.L. Nielsen*, C.F. Niordson \\ Department of Mechanical Engineering, Solid Mechanics, Technical University of Denmark, \\ DK-2800 Kgs. Lyngby, Denmark
}

\section{Abstract}

A single material length parameter governs both the material size dependence and the predicted micro-structural behavior in most existing micro-structurally based continuum theories. As a consequence, smoothly varying field quantities are predicted which contrast recent years experimental observations. In a previous work by the authors, addressing this matter, two new back stress formulations were proposed, and demonstrated to offer novel modeling capabilities in the localization behavior of geometrically necessary dislocation pile-up under monotonic loading. However, the cyclic behavior of these formulations remains to be investigated. The present work studies the new back stress formulations, within a non-work conjugate type higher order strain gradient crystal plasticity framework, and demonstrates their performance through the idealized single slip simple shear case. At high values of the material length scale parameter, a seemingly anomalous cyclic response is observed when deviating from the conventional type back stress formulation. Similar observations have recently been reported in other numerical studies, and the present work offers a discussion of the physical justification of such material behavior. It is found that the properties of the adopted formulations, in fact, open the possibility for modeling complex material behavior, tied to the pres-

\footnotetext{
${ }^{*}$ Tel: +45 4525-4258, Fax: +45 4593-1475

URL: kin@mek.dtu.dk (K.L. Nielsen)
} 
ence of long range internal stresses due to dislocation pile-up. Moreover, the present study extends the discussion on micro-structure predictions as a consequence of the adopted back stress models.

Keywords: Strain gradient plasticity, Size-effects, Bauschinger effect, Geometrically necessary dislocations, Crystal plasticity

\section{Introduction}

Through substantial experimental evidence, it is now well established that ductile crystalline materials exhibit size-dependent behavior in the presence of spatial gradients of plastic strain (see e.g. Fleck et al., 1994; Mu et al., 2014). More specifically, an increase in hardening and/or an apparent increase in yield point (also referred to as strengthening) is observed. The phenomena can be explained in terms of the so-called geometrically necessary dislocations (GNDs), and statistically stored dislocations (SSDs), of which the former play a key role in micron and sub-micron scale plasticity problems. One example, often referred to in the literature, is crack tip plasticity and its influence on the fracture toughness (e.g. Wei and Hutchinson, 1997, 1999; Nielsen et al., 2012; Martínez-Pañeda and Niordson, 2016). For several decades, gradient effects have been studied and numerous theories have been proposed to model the observed size effects. Within the class of continuum models both so-called lower order theories (e.g Bassani, 2001; Huang et al., 2004) and higher order theories have emerged. It is now generally accepted that higher order theories, which incorporate micro-structural boundary conditions, offer important modeling capabilities. Among the significant contributions are Fleck et al. (1994), Gurtin (2000, 2002), Fleck and Hutchinson (2001), Yefimov et al. (2004), Evers et al. (2004), Bayley et al. (2006), and Fleck and Willis (2009a,b). These 
theories allow boundary conditions on plastic strain quantities or associated stress quantities, which enter either through an extended virtual work principle or through additional partial differential equations. The continuum theories have, for many cases, been proven to adequately capture the size-dependent macroscopic behavior. However, common for these theories is the tendency to predict a rather smooth micro-structure, which is not consistent with experimental studies of metals (see also the recent discussion in El-Naaman et al., 2016).

It is well known that, during plastic deformation, dislocations tend to arrange in wall and cell structures within grains of crystalline materials, where bands of high dislocation density (dislocation walls) typically enclose regions of relatively low dislocation density (cell interiors) (see e.g. Ananthan et al., 1991; Huang and Winther, 2007; Hong et al., 2013). Öztop (2011) reported the same phenomenon for GNDs, in a nickel single crystal subject to wedge indentation, measured using high resolution electron backscatter diffraction (HR-EBSD) microscopy ${ }^{1}$. By linking the lattice curvature to dislocation density, through the Nye (1953) tensor, Öztop (2011) revealed distinct and highly localized GND wall and cell structures. A continuum description of this type of micro-structural behavior, suitable for large scale plasticity problems, remains to be fully established, despite the fact that this gap in the literature has received significant attention in the recent years (e.g. Klusemann and Yalçinkaya, 2013; van Beers et al., 2013, 2015; El-Naaman et al., 2016).

The micro-structural behavior is of particular interest in the present study, where

\footnotetext{
${ }^{1}$ Details on the experimental methods employed by Öztop (2011) can also be found in Kysar et al. (2010) and Dahlberg et al. (2014), also confirming the existence of highly localized GND structures.
} 
the non-work conjugate type formulation, treated by Kuroda and Tvergaard (2006, 2008), is adopted. The virtual work principle remains the conventional one in this type of theory, while the evolution of GND densities is accounted for through additional differential equations. In this continuum theory, a back stress, related to the micro-structure, accounts for the gradient effects in the plastic behavior, and the mathematical form of such a back stress is still an open matter. However, back stress models can, for the most part, be classified into two types: i) The physically based models, which build on considerations of the stress field of a single dislocation (e.g. Groma et al., 2003; Yefimov et al., 2004), and ii) the phenomenologically based models, which either build on a postulated strain gradient energy relation (e.g. Kuroda and Tvergaard, 2008; Öztop et al., 2012) or formulated directly to possess certain constitutive properties as in El-Naaman et al. (2016).

In an attempt to develop suitable back stress formulations, that allow for nonsmooth evolution of dislocation micro-structure, two candidates were proposed and studied under monotonic loading in El-Naaman et al. (2016). However, their behavior under cyclic loading remains unexplored despite being highly relevant for this type of model, where the Bauschinger effect is pivotal. In the following study advantages and characteristics of these back stress formulations will be identified and the resulting predictions of micro-structure will be discussed. To this end, attention will be confined to the representation of a single dislocation cell in the idealized simple shear case, which will serve as a lucid benchmark for the performance of the models proposed in El-Naaman et al. (2016).

The paper is structured as follows. The strain gradient plasticity model is described 
in Section 2, and the two back stress formulations treated are briefly outlined in Section 3 (more details can be found in El-Naaman et al., 2016). The boundary value problem is described in Section 4, after which a series of results are presented in Section 5, using a finite element method (FEM) implementation. The cyclic response is discussed in Section 6, and the final concluding remarks are given in Section 7.

\section{Strain gradient crystal plasticity model}

The present study employs a small strain formulation, with the total strain rate given by $\dot{\varepsilon}_{i j}=\left(\dot{u}_{i, j}+\dot{u}_{j, i}\right) / 2$, which decomposes into an elastic part, $\dot{\varepsilon}_{i j}^{e}$, and a plastic part, $\dot{\varepsilon}_{i j}^{p}$, so that; $\dot{\varepsilon}_{i j}=\dot{\varepsilon}_{i j}^{e}+\dot{\varepsilon}_{i j}^{p}$. The formulation builds on the framework of conventional crystal visco-plasticity theory, where plastic deformation occurs by means of crystallographic slip on the individual slip systems. Thus, the plastic strain rate is given in terms of the slip rate, $\dot{\gamma}$, on the $\alpha^{\prime}$ th slip system, as

$$
\dot{\varepsilon}_{i j}^{p}=\sum_{\alpha} \dot{\gamma}^{(\alpha)} P_{i j}^{(\alpha)}, \quad P_{i j}^{(\alpha)}=\frac{1}{2}\left(s_{i}^{(\alpha)} m_{j}^{(\alpha)}+m_{i}^{(\alpha)} s_{j}^{(\alpha)}\right),
$$

where the superposed dot denotes material time derivative, $P_{i j}^{(\alpha)}$ is the Schmid orientation tensor, and $s_{i}^{(\alpha)}$ and $m_{i}^{(\alpha)}$ are unit vectors specifying the slip direction and slip plane normal, respectively (see also Fig. 1b for a schematic definition). The slip rate is given by

$$
\dot{\gamma}^{(\alpha)}=\dot{\gamma}_{0} \operatorname{sgn}\left(\tau^{(\alpha)}-\tau_{b}^{(\alpha)}\right)\left(\frac{\left|\tau^{(\alpha)}-\tau_{b}^{(\alpha)}\right|}{g^{(\alpha)}}\right)^{1 / m},
$$

where $\dot{\gamma}_{0}$ is a reference slip rate, $\tau^{(\alpha)}$ is the Schmid stress (taken as the macroscopic Cauchy stress tensor resolved onto the slip plane), $\tau_{b}^{(\alpha)}$ is a back stress, $m$ is the rate sensitivity exponent, and $g^{(\alpha)}$ is the slip resistance. The hardening law is taken as

$$
\dot{g}^{(\alpha)}=\sum_{\beta} h^{(\alpha \beta)}\left|\dot{\gamma}^{(\beta)}\right|,\left.\quad g^{(\alpha)}\right|_{t=0}=\tau_{0}
$$


where the initial value of $g^{(\alpha)}$ is chosen as the initial critical resolved shear stress, $\tau_{0}$. Here, $t$ is time and $h^{(\alpha \beta)}$ are slip hardening moduli, in which the trace accounts for self hardening, and the off-diagonal parts for latent hardening. Equation (2) is a gradient-enhanced version of the widely used conventional visco-plastic power law slip rate relation (Hutchinson, 1976; Peirce et al., 1983), in that $\tau^{(\alpha)}-\tau_{b}^{(\alpha)}$ replaces $\tau^{(\alpha)}$ as an effective resolved shear stress ${ }^{2}$. The back stress, $\tau_{b}^{(\alpha)}$, is related directly to the gradients of the GND density (see Section 3), and impedes (or aids, depending on load path) slip at non-uniform pile-ups of GNDs. Thereby, the effective resolved shear stress becomes the driving force behind dislocation glide (Harder, 1999; Groma et al., 2003; Yefimov et al., 2004; Evers et al., 2004).

A non-work conjugate type higher order strain gradient plasticity theory is employed in the present study. This theory, formulated by Kuroda and Tvergaard (2006, 2008), is of a higher order nature but does not employ higher order stresses work conjugate to strain gradients or higher order equilibrium equations. Instead, the governing equations of the non-work conjugate formulation, are given by the conventional stress equilibrium equation; $\sigma_{i j, j}+f_{i}=0$, where $f_{i}$ are body forces and the Cauchy stress rate tensor is given by the elastic relationship; $\dot{\sigma}_{i j}=\mathscr{L}_{i j k l}\left(\dot{\varepsilon}_{k l}-\dot{\varepsilon}_{k l}^{p}\right)$, in which $\mathscr{L}_{i j k l}$ is the fourth order elastic stiffness tensor. The conventional rate-dependent incremental principle of virtual work thereby reads:

$$
\int_{V} \mathscr{L}_{i j k l} \dot{\varepsilon}_{k l} \delta \dot{\varepsilon}_{i j} \mathrm{~d} V=\int_{V} \mathscr{L}_{i j k l} \dot{\varepsilon}_{k l}^{p} \delta \dot{\varepsilon}_{i j} \mathrm{~d} V+\int_{V} \dot{f}_{i} \delta \dot{u}_{i} \mathrm{~d} V+\int_{S} \dot{T}_{i} \delta \dot{u}_{i} \mathrm{~d} S, \quad \dot{T}_{i} \equiv \dot{\sigma}_{i j} n_{j}
$$

where $\dot{T}_{i}$ is the traction rate, $n_{i}$ is the outward unit normal to the surface $S$ bounding the volume $V$. The evolution of GND density, in balance with the slip gradients, is

\footnotetext{
${ }^{2}$ Note that for $\tau_{b}^{(\alpha)}$ equal to zero, Eq. (2) reduces to the conventional theory.
} 
accounted for by an additional set of partial differential equations:

$$
\begin{aligned}
& \frac{1}{b} \gamma_{, i}^{(\alpha)} s_{i}^{(\alpha)}+\rho_{G(e)}^{(\alpha)}=0 \\
& \frac{1}{b} \gamma_{, i}^{(\alpha)} p_{i}^{(\alpha)}+\rho_{G(s)}^{(\alpha)}=0
\end{aligned}
$$

where $\mathbf{p}^{(\alpha)}=\mathbf{s}^{(\alpha)} \times \mathbf{m}^{(\alpha)}, b$ is the magnitude of the Burgers vector, and $\rho_{G}^{(\alpha)}$ are GND densities on slip system $\alpha$ (Ashby, 1970). Subscripts $(e)$ and $(s)$ denote edge and screw components, respectively. The GND densities appear directly as free field variables in Eqs. (5a) and (5b), and their weak form reads:

$$
\begin{array}{ll}
\frac{1}{b} \int_{V} \delta \rho_{, i} s_{i}^{(\alpha)} \gamma^{(\alpha)} \mathrm{d} V=\frac{1}{b} \int_{S} \delta \rho \zeta^{(\alpha)} \mathrm{d} S+\int_{V} \delta \rho \rho_{G(e)}^{(\alpha)} \mathrm{d} V, & \zeta^{(\alpha)} \equiv \gamma^{(\alpha)} n_{i} s_{i}^{(\alpha)}, \\
\frac{1}{b} \int_{V} \delta \rho_{, i} p_{i}^{(\alpha)} \gamma^{(\alpha)} \mathrm{d} V=\frac{1}{b} \int_{S} \delta \rho \eta^{(\alpha)} \mathrm{d} S+\int_{V} \delta \rho \rho_{G(s)}^{(\alpha)} \mathrm{d} V, & \eta^{(\alpha)} \equiv \gamma^{(\alpha)} n_{i} p_{i}^{(\alpha)},
\end{array}
$$

where $\delta \rho$ is a weighting function (or virtual GND density). In the GND density equations, a hard interface impenetrable for dislocations is enforced by

$$
\begin{gathered}
\zeta^{(\alpha)} \equiv \gamma^{(\alpha)} n_{i} s_{i}^{(\alpha)}=0 \\
\eta^{(\alpha)} \equiv \gamma^{(\alpha)} n_{i} p_{i}^{(\alpha)}=0,
\end{gathered}
$$

whereas a free boundary, where dislocations can exit the body, corresponds to

$$
\rho_{G(e)}^{(\alpha)}=\rho_{G(s)}^{(\alpha)}=0
$$

For a detailed discussion on the micro-structural boundary conditions, the authors refer to Kuroda and Tvergaard (2008) where also the discretized finite element equations can be found.

The governing equations for the incremental displacement field and GND density fields are evaluated separately, but the separate fields are linked together by the back stresses, according to Eq. (2). Hence, it is the back stress that introduces the gradient effects into the material response of the adopted strain gradient plasticity model. 


\section{Back stress formulations}

In a non-uniform field of GNDs, arising due to a spatial variation of plastic strain gradients in ductile crystalline materials, a long range internal stress field emerges. This can be represented as a back stress in continuum models. In the framework adopted, the back stress influences the viscous slip rates, together with the resolved shear stresses on the slip systems. This gives rise to a kinematic hardening contribution, which accounts for the micro-structural effects on plastic flow. Here, the two back stress formulations proposed in El-Naaman et al. (2016) will be investigated under cyclic loading. These are; (i) a thermodynamically consistent formulation based on a generalized gradient energy potential, and (ii) a straightforward purely phenomenological formulation.

The present study is confined to plane strain deformation, and thus, all terms associated with screw dislocations vanish. The interaction between slip systems (such as general latent hardening) is omitted for clarity of the analysis.

\subsection{Thermodynamically consistent back stress formulation}

By assuming that the back stress is given by the divergence of a higher order stress, $\xi_{i}^{(\alpha)}$, as

$$
\tau_{b}^{(\alpha)} \equiv-\xi_{i, i}^{(\alpha)}
$$

the adopted theory is equivalent to the work conjugate theory of Gurtin $(2000,2002)$, as was shown by Kuroda and Tvergaard $(2006)^{3}$. This immediately follows from the micro-force balance equation; $\xi_{i, i}^{(\alpha)}+\tau^{(\alpha)}-\tau_{\text {eff }}^{(\alpha)}=0$, where the effective resolved shear

\footnotetext{
${ }^{3}$ Note that the term 'higher order stress' refers to the stress quantity which is work conjugate to strain gradients or slip gradients in other theories.
} 
stress is given by $\tau_{e f f}^{(\alpha)}=\tau^{(\alpha)}-\tau_{b}^{(\alpha)}$. The higher order stress is derived from the plastic part of the free energy, $\psi_{G}$, as

$$
\xi_{i}^{(\alpha)}=\frac{\partial \psi_{G}^{(\alpha)}}{\partial \gamma_{, i}^{(\alpha)}} .
$$

Note that, by adopting relation (9), the non-work conjugate theory takes a work conjugate form within a thermodynamically consistent framework, once a continuous differentiable constitutive relation for the gradient energy, $\psi_{G}$, has been defined. Here, the following generalized gradient energy potential will be assumed:

$$
\psi_{G}^{(\alpha)}=\frac{1}{\mu+1} \tau_{0} L^{\mu+1}\left|\gamma_{, i}^{(\alpha)} s_{i}^{(\alpha)}\right|^{\mu+1}
$$

where $L$ is a material length scale parameter, that enters for dimensional consistency (see also Bardella, 2010; Bardella and Panteghini, 2015; Fleck et al., 2015; El-Naaman et al., 2016). Note that, for the parameter interval of interest $(\mu>0)$, the free energy remains a convex function of the slip gradients.

The generalized energy expression in Eq. (11) leads to a back stress which is linear in the second order gradient of slip, but when $\mu \neq 1$ and $\mu>0$, includes a dependence on the first order gradient of slip (or the GND densities), to the power of $\mu-1$ (ElNaaman et al., 2016):

$$
\tau_{b}^{(\alpha)}=-\mu \tau_{0} L^{\mu+1}\left|\gamma_{, j}^{(\alpha)} s_{j}^{(\alpha)}\right|^{\mu-1} \gamma_{, k i}^{(\alpha)} s_{i}^{(\alpha)} s_{k}^{(\alpha)}
$$

Expressed in terms of GND densities, this relation becomes

$$
\tau_{b}^{(\alpha)}=\mu \tau_{0} b^{\mu} L^{\mu+1}\left|\rho_{G(e)}^{(\alpha)}\right|^{\mu-1} \rho_{G(e), i}^{(\alpha)} s_{i}^{(\alpha)}
$$

For $\mu=1$, Eq. (11) is quadratic and Eqs. (12) and (13) reduce to the more commonly used form of the back stress (see e.g. Kuroda and Tvergaard, 2008). 
Note that, due to the linear dependence on GND density gradients, the back stress in Eq. (13) does not give rise to long range internal stresses in a uniform field of GNDs. Equations (12) and (13) maintain thermodynamic consistency through Eq. (9), while, in the wording of Kuroda and Tvergaard (2006), they have a non-constant back stress coefficient, which evolves with the GND density for $\mu \neq 1$.

For $0<\mu<1$, the back stress in Eq. (13) is singular when the GND density goes to zero, and thus, an "effective" GND density is employed, such that; $\rho_{G(e), e f f}^{(\alpha)}=\left|\rho_{G(e)}^{(\alpha)}\right|+\rho_{0}$ replaces $\left|\rho_{G(e)}^{(\alpha)}\right|$ in Eq. (13), for values of $\mu$ below one. This is a numerical necessity to facilitate a stable solution for low GND densities, but it essentially resembles the presence of SSDs in the back stress relation as in e.g. Groma et al. (2003) and Yefimov et al. (2004). A value of $\rho_{G(e), 0}=10^{11} \mathrm{~m}^{-2}$ is used in the present computations, unless otherwise stated, and a sensitivity analysis, performed in El-Naaman et al. (2016), showed $\rho_{0}$ to have relatively little influence on the model behavior ${ }^{4}$. Although this is true for low values of the material length scale (as studied in El-Naaman et al., 2016), the influence becomes significant when going to extreme values of $L$, as will be demonstrated in the present study.

For the evaluation of Eq. (13), it was chosen to use an element value of $\rho_{G(e)}^{(\alpha)}$, taken as an average of the nodal values, in order to increase numerical stability.

\subsection{Phenomenologically constructed back stress formulation}

As was shown in El-Naaman et al. (2016), slip localization is promoted through the relationship of the back stress with GND density, when $\mu \longrightarrow 0$ as well as for $\mu \geq 1$, when

\footnotetext{
${ }^{4}$ Note that in the paper El-Naaman et al. (2016), the unit measure for dislocation density was, by mistake, specified as $\left[\mathrm{m}^{-2}\right]$ throughout but was in fact $\left[\mathrm{mm}^{-2}\right]$.
} 
adopting Eqs. (12) and (13). This property can be attractive when modeling the highly non-uniform distribution of GNDs observed in experimental studies. In the framework proposed by Kuroda and Tvergaard $(2006,2008)$ any function $\tau_{b}^{(\alpha)}\left(\rho_{G(e), i}^{(\alpha)} s_{i}^{(\alpha)}\right)$ can, however, be considered, and the same type of localization behavior was also achieved for a back stress formulated as a function exclusively dependent on the GND density gradients (El-Naaman et al., 2015, 2016). This back stress is given by a piece-wise function, in which the first part follows from a quadratic free energy (through Eqs. (9) - (10)), and a parameter $\tau_{T}$ defines a transition point into a power law dependence on the GND density gradients:

$$
\tau_{b}^{(\alpha)}=\left\{\begin{array}{ll}
b \tau_{0} L^{2} \rho_{G(e), i}^{(\alpha)} s_{i}^{(\alpha)} & , \text { for }\left|\tau_{b}^{(\alpha)}\right| \leq \tau_{T} . \\
\operatorname{sgn}\left(\rho_{G(e), i}^{(\alpha)} s_{i}^{(\alpha)}\right) b^{\kappa} \tau_{T}^{1-\kappa} \tau_{0}^{\kappa} L^{2 \kappa}\left|\rho_{G(e), i}^{(\alpha)} s_{i}^{(\alpha)}\right|^{\kappa} & , \text { for }\left|\tau_{b}^{(\alpha)}\right|>\tau_{T}
\end{array} .\right.
$$

The first part of the piece-wise function excludes the initial infinite slope of the power law relation in Eq. (14), and it implies that the back stress evolves according to a quadratic free energy until a preassigned level $\left(\left|\tau_{b}^{(\alpha)}\right|=\tau_{T}\right)$, whereafter it transitions to the power law for $0<\kappa<1$. With $\kappa=0, \tau_{T}$ becomes a cut-off level. For the special case of $\kappa=1$, where a quadratic gradient energy prevails throughout, the model may be viewed as thermodynamically based. In all other cases, since a gradient energy relation that leads to Eq. (14) is not readily available, this back stress formulation must be considered a purely phenomenological formulation. However, all numerical solutions presented here, were found to satisfy positive dissipation throughout the load history for all values of $\kappa$, such that

$$
\sigma_{i j} \dot{\varepsilon}_{i j}^{p}=\sum_{\alpha} \tau^{(\alpha)} \dot{\gamma}^{(\alpha)} \geq 0
$$




\section{Boundary value problem}

The single slip simple shear problem, illustrated in Fig. 1, is considered in order to demonstrate the behavior of the back stress formulations under cyclic loading. In order to compare results to those found for monotonic loading in Kuroda and Tvergaard (2008) and El-Naaman et al. (2016), the following model parameters are used throughout: Slip angle $\theta=90^{\circ}$, Young's modulus $E=130 \mathrm{GPa}$, Poisson's ratio $\nu=0.3$, slip resistance $\tau_{0}=50 \mathrm{MPa}$, and Burgers vector $b=0.286 \mathrm{~nm}$. The material slab, of height $H$ and width $W$, is modeled as infinite in the $x_{1}$-direction by imposing the following periodic boundary conditions:

$$
\begin{aligned}
& \dot{u}_{i}\left(0, x_{2}\right)=\dot{u}_{i}\left(W, x_{2}\right) \\
& \rho_{G(e)}^{(\alpha)}\left(0, x_{2}\right)=\rho_{G(e)}^{(\alpha)}\left(W, x_{2}\right) .
\end{aligned}
$$

The additional boundary conditions imposed on the displacement field are

$$
\begin{aligned}
& \dot{u}_{1}=\dot{u}_{2}=0 \text { at } x_{2}=0 \\
& \dot{u}_{1}=\dot{U} \text { and } \dot{u}_{2}=0 \text { at } x_{2}=H,
\end{aligned}
$$

while the top and bottom boundaries of the material domain are microscopically passivated by the following higher order boundary conditions on the GND density field equations:

$$
\zeta^{(\alpha)} \equiv \gamma^{(\alpha)} n_{i} s_{i}^{(\alpha)}=0 \text { at } x_{2}=0 \text { and } x_{2}=H .
$$

The above listed boundary conditions imply that all fields become one dimensional. The results are obtained using the finite element method (FEM), solving the conventional and micro-structural equilibrium equations in a staggered scheme. Eight-node isoparametric quadrilateral elements with reduced Gauss integration are used for the 
displacement field analysis, whereas equivalent four-node elements with full Gauss integration are used for the GND density field analysis. Time integration is carried out by the forward Euler method. The finite element mesh used consists of 58 elements in the $x_{2}$-direction including a refined mesh at the microscopically passivated boundaries to resolve the steep gradients.

The procedure for determining the GND density field is independent of the specific back stress formulation analyzed, and in that sense, the framework has proved to be numerically robust. However, when the influence from the back stress becomes significant (e.g. achieved by increasing the length parameter), stability issues do arise in the subsequent determination of the associated incremental displacement field. This is independent of the employed back stress formulation and even the back stress originating from a quadratic free energy displays the same problem. The issues are related to the time stepping scheme employed and smaller time steps may improve stability for larger back stresses.

\section{Results}

The following numerical study focuses on the macroscopic response under cyclic loading, when employing the two different back stress formulations given by Eq. (13) and Eq. (14). Moreover, the predicted evolution in micro-structure is discussed in relation to the macroscopic behavior.

\subsection{Macroscopic response under cyclic loading}

At first, the boundary value problem described in Section 4 is solved with conventional hardening $\left(h^{(\alpha \beta)}=h=250 \mathrm{MPa}\right)$, at a rate sensitivity $m=0.02$. With 
$\dot{U}=H \dot{\gamma}_{0}$, the material domain is sheared to a macroscopic shear strain $\Gamma=0.03$ $\left(=U_{\max } / H\right)$, after which, one complete load cycle is performed, such that the macroscopic shear strain goes once to -0.03 and back to 0.03 . Figure 2 shows results obtained when adopting the thermodynamically consistent back stress formulation in Eq. (13) for different values of $\mu$. The complete shear stress vs. macroscopic shear strain curve is shown in Fig. $2 a^{5}$. For the final load step, the slip profile can be seen in Fig. $2 b$ and the distribution of GNDs can be seen in Fig. 2c. The GND density distributions in Fig. 2c are normalized with the values found at the boundary, denoted $\rho_{G(e), B}^{(1)}$, to enable qualitative comparison. Going from $\mu=1$ (quadratic free energy) to $\mu=0.4$ the spatial gradient of slip decreases, corresponding to a smoother distribution of GNDs (Fig. 2c), while a low value of $\mu$ (close to linear free energy) results in a steep gradient in slip, and thereby more localized pile-up of GNDs.

Along the same line, using the purely phenomenological back stress formulation in Eq. (14), similar localized GND structures are predicted in Fig. 3. For different values of $\kappa$ and with $\tau_{T} / \tau_{0}=0.12$, Fig. 3a shows the shear stress vs. macroscopic shear strain over the entire modeling history ${ }^{6}$. Figures $3 \mathrm{~b}$ and $3 \mathrm{c}$ show the slip profile and the GND density, respectively, after the final load step. When letting $\kappa$ go to zero, large slip gradients occur locally at the hard boundaries (Fig. 3b), resulting in increasingly localized GND structures (Fig. 3c), in contrast to the more smooth development seen for $\kappa=1$ (quadratic gradient energy). It is noticed that an additional wave appears in the central part of the slip profile for the low values of $\kappa$ (see Fig. 3b).

In the following, a parametric study, for the case of zero conventional hardening

\footnotetext{
${ }^{5}$ Note that the curves for $\mu=1$ and $\mu=1.1$ are overlapping.

${ }^{6}$ Note that the curves for $\kappa=0.05$ and $\kappa=0.1$ are partially overlapping
} 
$\left(h^{(\alpha \beta)}=h=0\right)$, is performed in order to bring out additional details on how the micro-structural predictions relate to the macroscopic response, considering the two different back stress formulations. In these computations it was checked that a steady state cyclic response was achieved by running multiple load cycles about the zero strain axis. Figure 4 shows the shear stress vs. macroscopic shear strain using the thermodynamically consistent back stress formulation in Eq. (13). Results are shown for different values of the back stress exponent, $\mu$ (Fig. 4a), for different values of the material length parameter, $L$ (Fig. $4 \mathrm{~b}$ ), and for different values of the additional microstructural parameter, $\rho_{0}$ (Fig. 4c). For the low material length parameter value $(L / H$ $=0.3)$ there is no considerable difference in the macroscopic response for $\mu=0.01$, 1.0, and 2.0 (the significance of this observation will be elaborated on in Section 5.2). However, when going to higher values of the material length parameter, a seemingly anomalous local change in slope on the response curves is predicted during plastic flow, so that these exhibit an inflection point close to zero strain (Fig. 4b). For the intermediate material length scale $(L / H=5)$, Fig. 4c clearly shows that this behavior is largely controlled by the micro-structural parameter, $\rho_{0}$ (see discussion in Section $6)$.

Figure 5 shows the shear stress vs. macroscopic shear strain using the purely phenomenologically constructed back stress formulation in Eq. (13). Results are shown for different values of the back stress exponent, $\kappa$ (Fig. 5a), for different material length scales, $L$ (Fig. 5b), and for different values of the transition parameter, $\tau_{T}$ (Fig. 5c). Similarly, Fig. 5a shows no significant difference in the macroscopic response for the low value of the material length parameter $(L / H=0.3)$, when varying the value of $\kappa$ (see 
also Section 5.2). Again, similar to the predictions resulting from the former back stress formulation, when going to higher material length scales, an anomalous local change in slope on the response curves is seen during plastic flow, with an inflection point close to zero strain (Fig. 5b). Figure 5c shows that this behavior is largely controlled by the transition parameter, $\tau_{T}$. For the thermodynamically consistent formulation, this local slope change takes place rather smoothly, whereas the purely phenomenological formulation displays a much more rapid change in slope. This is due to the particular choice of constitutive parameters, which will be elaborated in the following (see also Section 6).

To understand what controls this seemingly anomalous cyclic behavior observed for both back stress formulations, the plastic slip and back stress distribution are plotted across the material slab height at different stages of the load cycle (Figs. 6 through 10). The different load stages are marked and numbered on one representative curve in each of Figs. 4b and 5b. Figure 6 shows results for a back stress based on quadratic free energy $(\mu=\kappa=1)$, while Figs. 7 and 8 show results using back stress relation in Eq. (13) with $\mu=0.01$ and back stress relation in Eq. (14) with $\kappa=0.1$, respectively. In these computations, a low value of the material length parameter $(L / H=0.3)$ is used. For the phenomenological back stress formulation (Eq. (14)), the value $\tau_{T} / \tau_{0}=$ 0.06 will be used henceforth. It is generally observed that, when the load is reversed, the back stress changes direction over a gradually increasing domain (illustrated by stages 1 through 6 ), and that the change initiates locally at the micro-structurally passivated boundaries. A large central portion of the slab height experiences relatively little change in back stress (see Figs. 6-8). This is especially true when assuming a cubic 
energy potential $(\mu=2.0)$, where the central part remains basically unchanged with zero back stress throughout the deformation (Fig. $7 d)^{7}$. Furthermore, it is noticed that for the purely phenomenological formulation, the majority of the material is dominated by a quadratic free energy, which is illustrated by the $\left|\tau_{b}^{(1)}\right|=\tau_{T}$ lines plotted in Fig. 8b. Thus, the power law part of Eq. (14) controls only the outermost regions of the material domain close to the passivated boundaries.

In Figs. 9 and 10, where a high material length scale is employed $(L=10)$, it is observed that a high level of back stress develops in the entire material domain (see Figs. 9b and 10b). Thus, the behavior of the back stress controls, quite directly, the macroscopic response throughout, resulting in the pronounced unconventional Bauschinger effect, in terms of inflection points. The change in back stress initiates at the boundaries here as well, but subsequently the shift from aiding slip to impeding slip takes place in the entire domain upon reversed loading. Moreover, it is noticed in Fig. 10b, that the power law part of Eq. (14) dominates the material completely in stages 1, 2, 5, and 6. The macroscopic behavior of the models is further discussed in Section 6 .

\subsection{Micro-structure evolution predictions}

In general, dislocations tend to form cell structures in metals under plastic deformation and the same behavior has been reported for the evolution of GNDs. The following results confirm the fact that the approaches taken in El-Naaman et al. (2016), to the formulation of the back stress, offer additional control of the micro-structural predictions mainly through one additional model parameter ( $\mu$ or $\kappa$ in the two models,

\footnotetext{
${ }^{7}$ It is noted that some asymmetries appear for $\mu=2$, which is especially clear when comparing stage 1 and stage 6 in Fig. 7c.
} 
respectively). As will become evident, this additional modeling capability is useful when seeking realistic micro-structures, and cannot be achieved in the classical type of back stress formulation (corresponding to $\mu=\kappa=1$ ), where the material length scale parameter, $L$, alone governs the micro-structural behavior.

Figures 11 and 12 show that there is a significant difference in the micro-structure predicted when deviating from the classical back stress formulation based on quadratic free energy $(\mu=\kappa=1)$. The figures show the GND density normalized with the value at the boundary, $\rho_{G(e), B}^{(1)}$, at $\Gamma=0.03$ after the final load step. Results are shown for different values of $\mu$ and $\kappa$, respectively. Figure 11 shows that, compared to a quadratic free energy, a more localized pile up of GNDs is predicted near the micro-structurally passivated boundaries, when approaching a linear gradient energy $(\mu=0.01,0.03)$. Similarly, when assuming a cubic free energy $(\mu=2)$, localization of GNDs is achieved at the boundaries. However, clear qualitative differences are observed here, in that dislocations of opposite sign appear to pile up adjacent to the dislocation walls at the passivated boundaries. For the purely phenomenological formulation a clearly localized micro-structure is achieved with a low power law dependence $(\kappa=0.1)$ compared to the traditional quadratic free energy assumption.

The results outlined above should be seen in contrast with the shear stress vs. macroscopic shear strain curves in Fig. 4a for the thermodynamically consistent formulation (for quadratic free energy, $\mu=1$, near linear free energy, $\mu=0.01,0.03$, and cubic free energy, $\mu=2$ ) and in Fig. 5a for the phenomenological formulation (for two choices of exponent, $\kappa=0.1$ and 0.4 , and quadratic free energy, $\kappa=1.0)$. In all cases the macroscopic response is nearly identical, while the predicted microstructure 
is largely different.

\section{Discussion of the inflected Bauschinger effect}

Cyclic plastic deformation of ductile crystalline materials gives rise to the Bauschinger effect, which reflects micro-structural mechanisms related to the pile up of dislocations. Compared to the findings in the present study, however, the Bauschinger effect is usually taken to proceed more smoothly or gradually, and not cause inflection points such as those seen in Figs. 4 and 5. The inflection points on the flow curves express a sudden increase and subsequent decrease in growth rate of the back stress close to the zero strain point. This causes a shift in the flow stress level and, in some cases, gives the impression of two stages of plastic deformation. In terms of the thermodynamically consistent formulation (Eqs. (12) and (13)), the inflection points are essentially caused by the back stress approaching its singularity as the GND density goes to zero (in competition with the GND density gradient going to zero). How close the back stress can come to singular behavior is controlled by $\rho_{0}$, and thus, also the characteristics of the flow curve surrounding the inflection point is controlled by $\rho_{0}$ (see Fig. 4c). When the macroscopic shear strain changes sign, the growth rate of the back stress starts to decrease as it moves away from the singularity.

Interestingly, the very differently devised purely phenomenological back stress formulation (Eq. (14)) exhibits a behavior similar to that of the thermodynamically consistent back stress. Here, also showing a temporary change in slope of the flow curve, which initiates immediately after stage 4 (see Fig. 5) and persists approximately until stage 5 . Approximately at stage 4 , the back stress in the majority of the material domain has changed sign and is now impeding slip. Moreover, at this point 
and until stage 5, the quadratic free energy dominates (see Fig. 10b), and the slope of the inflection tangent is approximately that corresponding to quadratic free energy. Thus, for $\kappa=1$ (quadratic gradient energy throughout), the inflection tangent slope will persist throughout the plastic deformation. The appearance of the jump in the flow curve, in terms of strain interval and slope of the inflection tangent, is controlled by an interplay between $\tau_{T}$ and $L$ (see Figs. $5 \mathrm{~b}$ and $5 \mathrm{c}$ ). The rather pronounced inflection point, resulting from the present particular combination of $\kappa, \tau_{T}$, and $L$, may not be physically accurate, but emphasizes how this back stress relation works in practice.

Not only has the inflected type of Bauschinger effect been reported recently in other numerical strain gradient plasticity studies (Bardella and Panteghini, 2015; Wulfinghoff et al., 2015), but also experimental evidence of such cyclic behavior exists in the literature: Taillard and Pineau (1982) observed unusual inflected hysteresis curves in an overaged large grained alloy during the initial 20 cycles, which they attributed to long range internal stresses due to dislocation loops deposited around precipitatesin line with the preceding findings of Stoltz and Pelloux (1975), where the inflected Bauschinger effect was found to diminish or vanish with annealing treatments in alloys containing non-shearable precipitates. The governing mechanisms involved in these experimental studies, are analogous to the pile-up of dislocations at the passivated boundaries imposed in the present computations. Asaro (1975) also observed hysteresis curves with inflection points in the dispersion hardened super alloy In-753, which exhibits what they refer to as type III kinematic hardening, and it was stated that this type of hardening was also observed in a material that did not contain any substantial substructure aside from grain boundaries. Moreover, they showed that this behavior 
is a natural outcome of theories that incorporate hardening due to long range internal stresses. More recently, Proudhon et al. (2008) reported anomalous changes in slope upon reentry into plastic deformation in aluminum alloys.

At present, due to the lack of experimental data on cyclic plasticity in single crystal systems including passivated boundaries, it is unknown whether this type of macroscopic behavior can be observed in reality. However, since the inflected hysteretic behavior is believed to be tied to the influence of long range internal stresses due to pile-up of dislocations, which plays a significant role in micron-scale plasticity within the grains of metals, it is not reasonable to brush this type of behavior aside as nonphysical. A comparison to a dislocation dynamics simulation of the set-up outlined in Section 4 could shed more light on the phenomenon. However, this is outside the scope of this work.

\section{Concluding remarks}

A finite element implementation of the non-work conjugate higher order strain gradient crystal plasticity model, proposed by Kuroda and Tvergaard (2006, 2008), has formed the basis of an investigation of back stress formulations under cyclic loading. The back stress formulations used were proposed in El-Naaman et al. $(2015,2016)$ with the objective of improving the ability to predict micro-structural evolution. This paper treats the highly idealized pure shear case, which serves as a benchmark for the performance of the proposed model. The key findings of the present study can be summarized as follows:

i) Both employed back stress models perform well under cyclic loading, however, it is predicted that the hysteresis curves, in some cases, have inflection points, at 
around zero macroscopic shear strain, when deviating from the quadratic form of the free energy. This type of hysteretic behavior has substantial experimental backing, and is believed to be tied to the long range internal stresses due to dislocation pile-up and the reversibility of these during backward straining (Asaro, 1975). The characteristics of the inflected Bauschinger effect, is controlled by the parameters $L, \mu, \rho_{0}, \kappa$, and $\tau_{T}$, in the two models, respectively, and thus, the additional model parameters offer the possibility to model this particular material behavior reported in the literature. Moreover, the authors speculate that these properties can be utilized to capture the micro-structural heterogeneity and discontinuities of real ductile crystalline materials.

ii) It is demonstrated that with both back stress formulations it is possible to exert some level of control of the micro-structural predictions of the model, and to some extent, independently of the macroscopic response. This is achieved mainly through the additional model parameter denoted $\mu$ or $\kappa$ in the two models, respectively.

With the present cyclic investigation, the performance of the two adopted back stress formulations is further established, both in terms of the macroscopic response but also in relation to the predicted dislocation micro-structure. However, it must be emphasized that further research on more complicated boundary value problems, e.g. including multi-slip and latent hardening, is needed in order to fully establish the applicability of the back stress formulations proposed in El-Naaman et al. (2016). 


\section{Acknowledgments}

The work is financially supported by The Danish Council for Independent Research under the research career programme Sapere Aude as part of the project "Higher Order Theories in Solid Mechanics", grant 11-105098/FTP.

\section{References}

Ananthan, V., Leffers, T., Hansen, N., 1991. Cell and band structures in cold-rolled polycrystalline copper. Materials Science and Technology 7, 1069-1075.

Asaro, R., 1975. Elastic-plastic memory and kinematic-type hardening. Acta Metallurgica $23,1255-1265$.

Ashby, M., 1970. The deformation of plastically non-homogeneous alloys. Philos. Mag. $21,399-424$.

Bardella, L., 2010. Size effects in phenomenological strain gradient plasticity constitutively involving the plastic spin. International Journal of Engineering Science 48, $550-568$.

Bardella, L., Panteghini, A., 2015. Modelling the torsion of thin metal wires by distortion gradient plasticity. Journal of the Mechanics and Physics of Solids 78, 467-492.

Bassani, J., 2001. Incompressibility and a simple gradient theory of plasticity. Journal of the Mechanics and Physics of Solids 49, 1983-1996.

Bayley, C., Brekelmans, W., Geers, M., 2006. A comparison of dislocation induced back stress formulations in strain gradient crystal plasticty. International Journal of Solids and Structures 43, 7268-7286. 
Dahlberg, C., Saito, Y., Öztop, M., Kysar, J., 2014. Geometrically necessary dislocation density measurements associated with different angles of indentations. International Journal of Plasticity 54, 81-95.

El-Naaman, S., Nielsen, K., Niordson, C., 2015. Strain gradient crystal plasticity: A continuum mechanics approach to modeling micro-structural evolution. Plastic Behavior of Conventional and Advanced Materials: Theory, Experiment, and Modeling, $7-9$.

El-Naaman, S., Nielsen, K., Niordson, C., 2016. On modeling micro-structural evolution using a higher order strain gradient continuum theory. International Journal of Plasticity 76, 285-298.

Evers, L., Brekelmans, W., Geers, M., 2004. Non-local cystal plasticity model with intrinsic SSD and GND effects. Journal of the Mechanics and Physics of Solids 52, 2379-2401.

Fleck, N., Hutchinson, J., 2001. A reformulation of strain gradient plasticity. Journal of the Mechanics and Physics of Solids 49, 2245-2271.

Fleck, N., Hutchinson, J., Willis, J., 2015. Guidelines for constructing strain gradient plasticity theories. Journal of Applied Mechanics 82, 1-10.

Fleck, N., Muller, G., Ashby, M., Hutchinson, J., 1994. Strain gradient plasticity: Theory and experiment. Acta Metallurgica et materialia 42 (2), 475-487.

Fleck, N., Willis, J., 2009a. A mathematical basis for strain-gradient plasticity theory. 
Part I: Scalar plastic multiplier. Journal of the Mechanics and Physics of Solids 57, $161-177$.

Fleck, N., Willis, J., 2009b. A mathematical basis for strain-gradient plasticity theory. Part II: Tensorial plastic multiplier. Journal of the Mechanics and Physics of Solids $57,1045-1057$.

Groma, I., Csikor, F., Zaizer, M., 2003. Spatial correlations and higher-order gradient terms in a continuum description of dislocation dynamics. Acta Materialia 51, 12711281.

Gurtin, M., 2000. On the plasticity of single crystals: free energy, microforces, plasticstrain gradients. Journal of the Mechanics and Physics of Solids 48 (5), 989-1036.

Gurtin, M., 2002. A gradient theory of single-crystal viscoplasticity that accounts for geometrically necessary dislocations. Journal of the Mechanics and Physics of Solids $50,5-32$

Harder, J., 1999. A crystallographic model for the study of local deformation processes in polycrystals. International Journal of Plasticity 15, 605-624.

Hong, C., Huang, X., Winter, G., 2013. Dislocation content of geometrically necessary boundaries aligned with slip planes in rolled aluminium. Philosophical Magazine 93 (23), 3118-3141.

Huang, X., Winther, G., 2007. Dislocation structures. Part I. Grain orientation dependence. Philosophical Magazine 87 (33), 5189-5214. 
Huang, Y., Qu, S., Hwang, K., Li, M., Gao, H., 2004. A conventional theory of mechanism-based strain gradient plasticity. International Journal of Plasticity 20, $753-782$.

Hutchinson, J., 1976. Bounds and self-consistent estimates for creep of polycrystalline materials. Proc. R. Soc. Lond. A 348, 101-127.

Klusemann, B., Yalçinkaya, T., 2013. Plastic deformation induced microstructure evolution through gradient enhanced crystal plasticity based on a non-convex Helmholtz energy. International Journal of Plasticity 48, 168-188.

Kuroda, M., Tvergaard, V., 2006. Studies of scale dependent crystal viscoplasticity models. Journal of the Mechanics and Physics of Solids 54, 1789-1810.

Kuroda, M., Tvergaard, V., 2008. On the formulation of higher-order strain gradient crystal plasticity models. Journal of the Mechanics and Physics of Solids 56, 15911608.

Kysar, J., Saito, Y., Oztop, M., Lee, D., Huh, W., 2010. Experimental lower bounds on geometrically necessary dislocation density. International Journal of Plasticity 26, $1097-1123$.

Martínez-Pañeda, E., Niordson, C., 2016. On fracture in finite strain gradient plasticity. International Journal of Plasticity, 154-167.

Mu, Y., Hutchinson, J., Meng, W., 2014. Micro-pillar measurements of plasticity in confined cu thin films. Extreme Mechanics Letters 1, 62-69. 
Nielsen, K., Niordson, C., Hutchinson, J., 2012. Strain gradient effects on steady state crack growth in rate-sensitive materials. Engineering Fracture Mechanics 96, 61-71.

Nye, J., 1953. Some geometrical relations in dislocated crystals. Acta Metallurgica $1(2), 153-162$.

Öztop, M., 2011. Multiscale experimental analysis in plasticity: Linking dislocation structures to continuum fields. Ph.D. thesis, Columbia University.

Öztop, M., Niordson, C., Kysar, J., 2012. Length-scale effect due to periodic variation of geometrically nescessary dislocaion densities. International Journal of Plasticity 41, 189-201.

Peirce, D., Asaro, R., Needleman, A., 1983. Material rate dependence and localized deformation in crystalline solids. Acta Metall. 31, 1951-1976.

Proudhon, H., Poole, W., Wang, X., Bréchet, Y., 2008. The role of internal stresses on the plastic deformation of the Al-Mg-Si-Cu alloy AA611. Philosophical Magazine $88,621-640$

Stoltz, R., Pelloux, R., 1975. The Bauschinger effect in precipitation strengthened aluminum alloys. Metallugical Transactions A 7 (8), 1295-1306.

Taillard, R., Pineau, A., 1982. Room temperature tensile properties of Fe-19wt.\%Cr alloys precipitation hardened by the intermetallic compound NiAl. Materials Science and Engineering 56, 219-231.

van Beers, P., McShane, G., Kouznetsova, V., Geers, M., 2013. Grain boundary in- 
terface mechanics in strain gradient crystal plasticity. Journal of the Mechanics and Physics of Solids 61, 2659-2679.

van Beers, R., Kouznetsova, V., Geers, M., 2015. Defect redistribution within a continuum grain boundary plasticity model. Journal of the Mechanics and Physics of Solids 83, 243-262.

Wei, Y., Hutchinson, J., 1997. Steady-State crack growth and work of fracture for solids characterized by strain gradient plasticity. Journal of the Mechanics and Physics of Solids 45, 1253-1273.

Wei, Y., Hutchinson, J., 1999. Models of interface separation accompanied by plastic dissipation at multiple scales. International Journal of Fracture 95, 1-17.

Wulfinghoff, S., Forest, S., Böhlke, 2015. Strain gradient plasticity modeling of the cyclic behavior of laminate microstructures. Journal of the Mechanics and Physics of Solids 79, 1-20.

Yefimov, S., Groma, I., van der Giessen, E., 2004. A comparison of a statisticalmechanics based plasticity model with descrete dislocation plasticity calculations. Journal of the Mechanics and Physics of Solids 52, 279-300. 


\section{Figures}

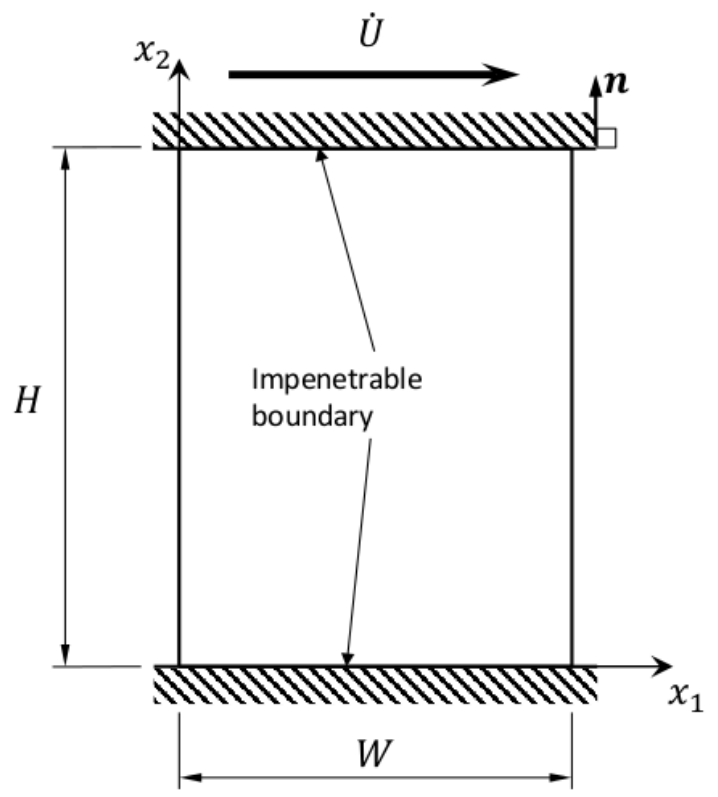

(a)

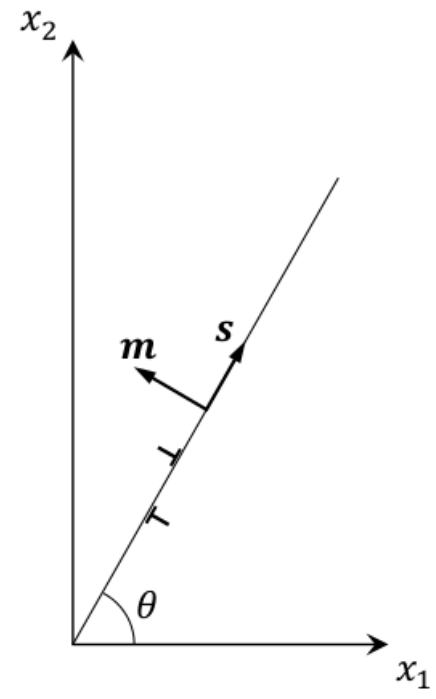

(b)

Figure 1: Schematic of the simple shear problem: (a) the material domain, which is modeled as infinite in the $x_{2}$-direction, is constrained by two rigid impenetrable boundaries and subject to a prescribed displacement rate $\dot{U}$, and (b) definition of the slip system. 


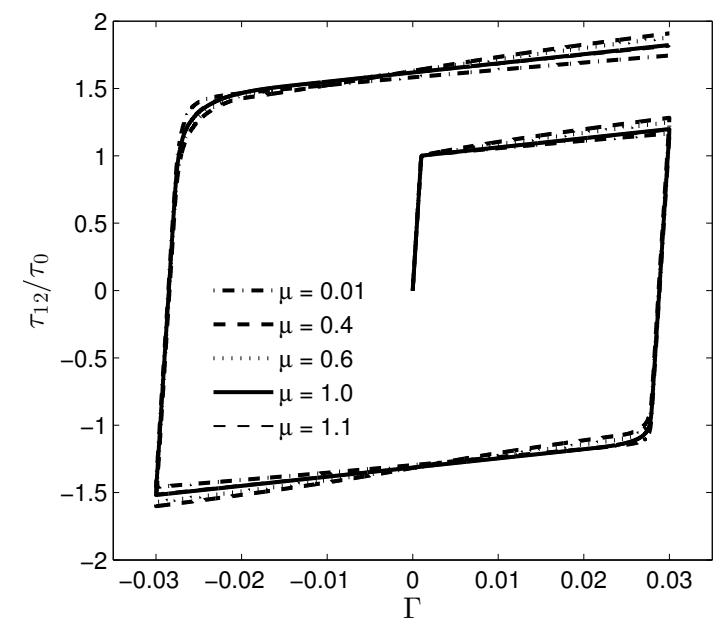

(a)

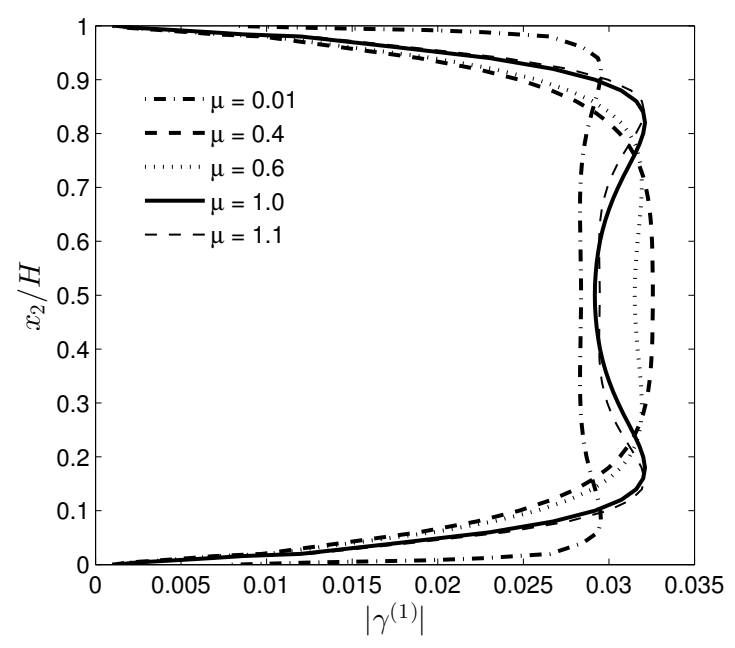

(b)

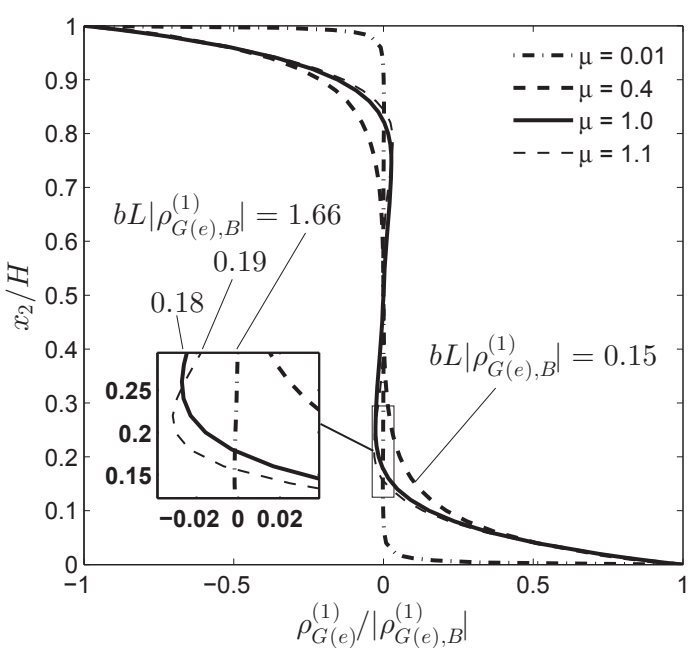

(c)

Figure 2: Numerical results for the single slip simple shear problem (Fig. 1) subject to one load cycle, using the generalized gradient energy based back stress (Eqs. (12) and (13)), with $\theta=90^{\circ}, h^{(\alpha \beta)}=h=250 \mathrm{MPa}, L / H=0.3, m=0.02, \rho_{0}=10^{11} \mathrm{~m}^{-2}$, and $\dot{U}=H \dot{\gamma}_{0}$, illustrating the effect of the $\mu$ parameter, on the cyclic behavior, in the linear to quadratic regime $(0<\mu \leq 1)$. The figure shows (a) shear stress vs. macroscopic shear strain, (b) slip profile, and (c) normalized GND density. Slip profile (b) and GND density (c) are shown for $\Gamma=0.03$ after the final load step. 


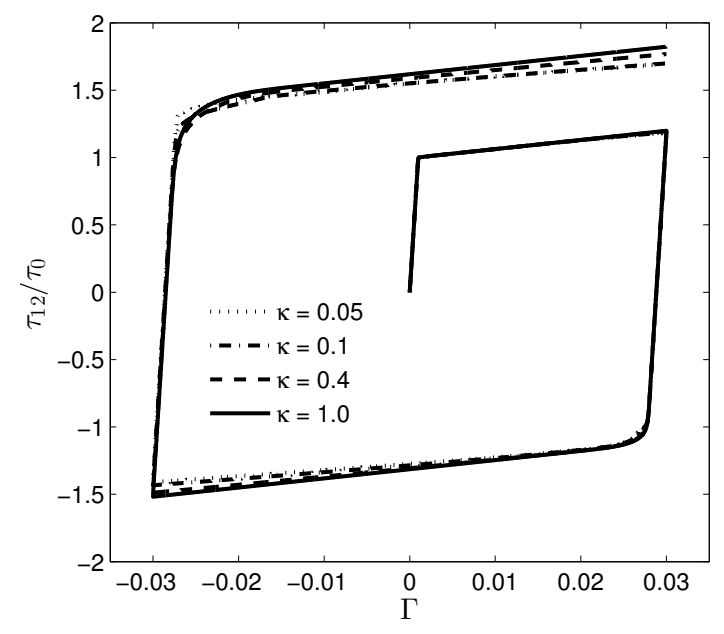

(a)

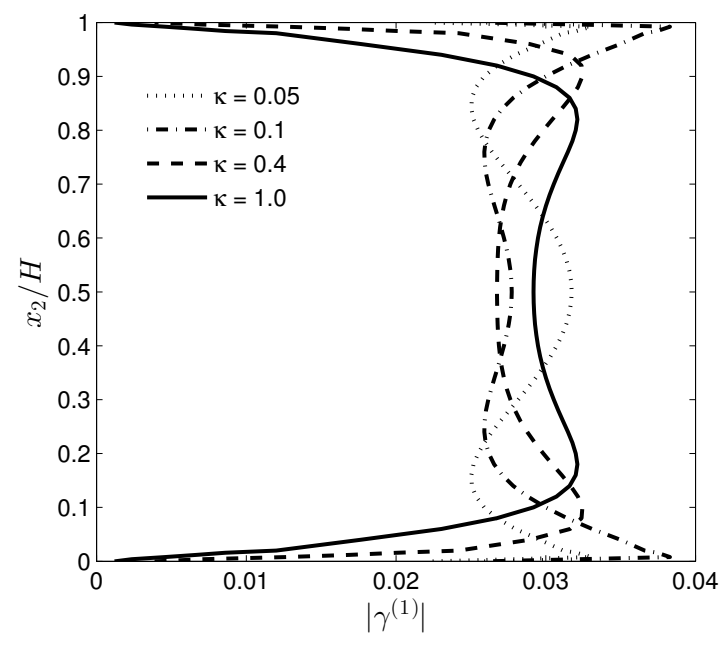

(b)

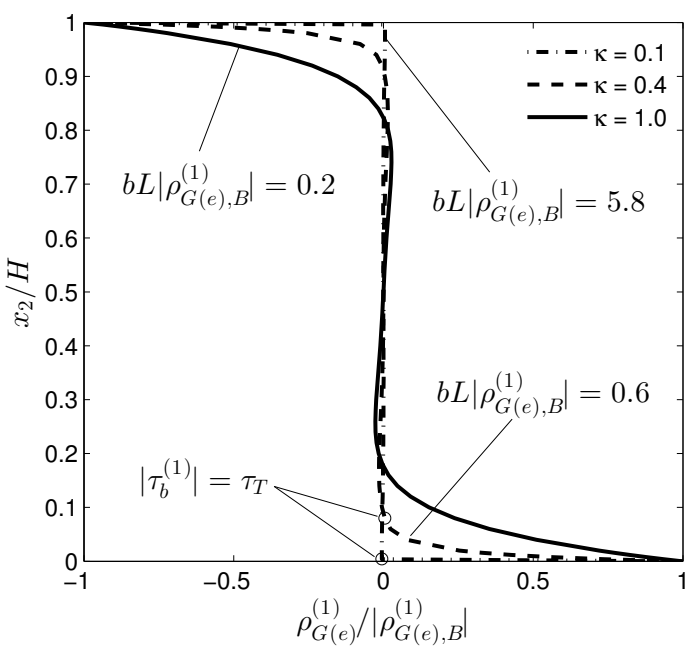

(c)

Figure 3: Shear stress vs. macroscopic shear strain for the single slip simple shear problem (Fig. 1) subject to one load cycle, using the back stress power law (Eq. (14)), with $\theta=90^{\circ}$, $h^{(\alpha \beta)}=h=250 \mathrm{MPa}, L / H=0.3, m=0.02, \tau_{T} / \tau_{0}=0.12$, and $\dot{U}=H \dot{\gamma}_{0}$, illustrating the effect of the $\kappa$ parameter on the cyclic behavior. The figure shows (a) shear stress vs. macroscopic shear strain, (b) Slip profile, and (c) normalized GND density, with circles marking the back stress transition point $\left(\left|\tau_{b}^{(1)}\right|=\tau_{T}\right)$ near the lower horizontal boundary. Slip profile (b) and GND density (c) are shown for $\Gamma=0.03$ after the final load step. 


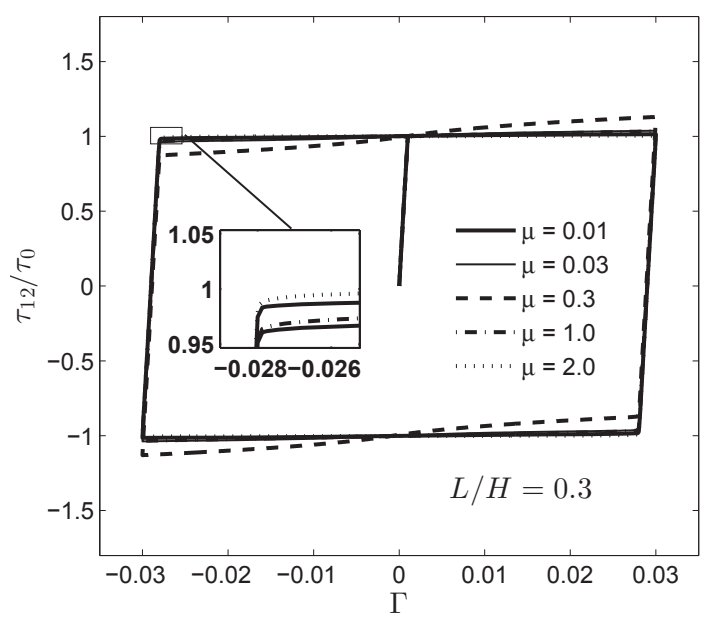

(a)

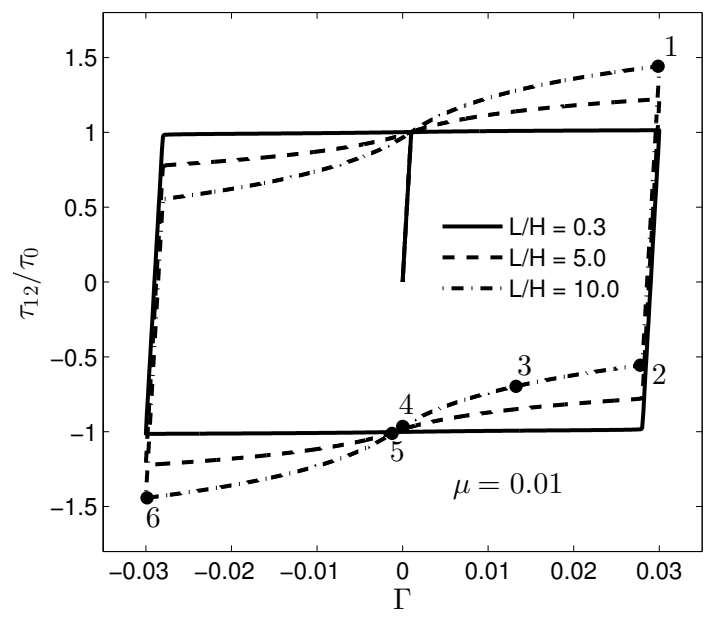

(b)

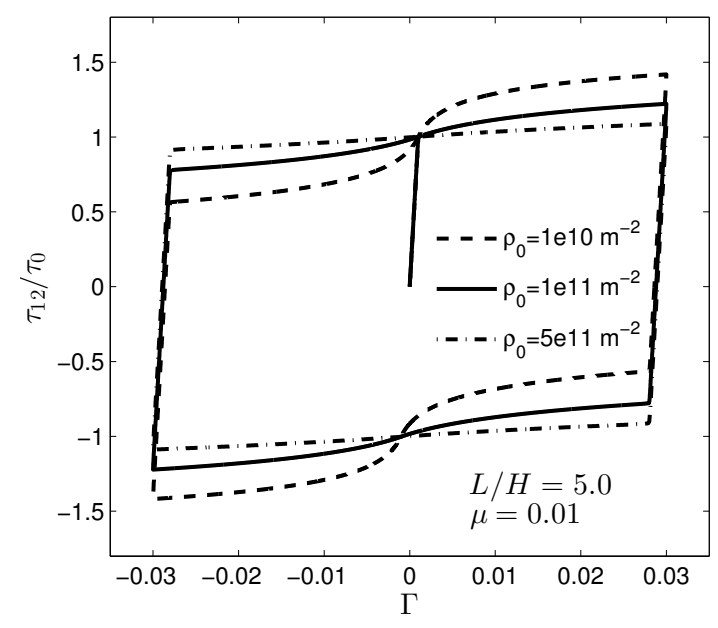

(c)

Figure 4: Numerical results for the single slip simple shear problem (Fig. 1), using the generalized gradient energy based back stress (Eqs. (12) and (13)), with $\theta=90^{\circ}, h^{(\alpha \beta)}=h$ $=0, m=0.02$, and $\dot{U}=H \dot{\gamma}_{0}$. The figure shows normalized shear stress vs. macroscopic shear strain: (a) for different values of $\mu$, (b) for different values of $L$, and (c) for different values of $\rho_{0}$. In (a) and (b), $\rho_{0}=1.0 \mathrm{e} 11 \mathrm{~m}^{-2}$ for $\mu<1$, and otherwise zero. In (b) the numbered dots mark the load stages at which the slip and back stress are plotted in the subsequent figures. 


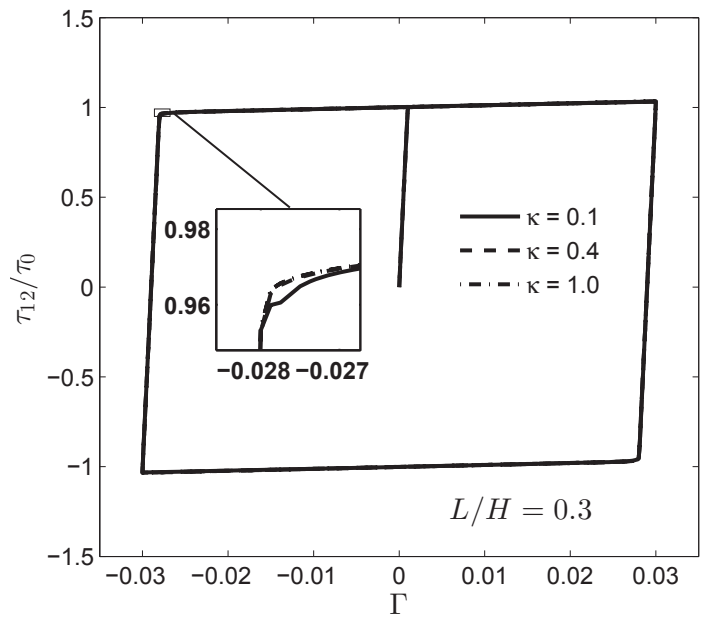

(a)

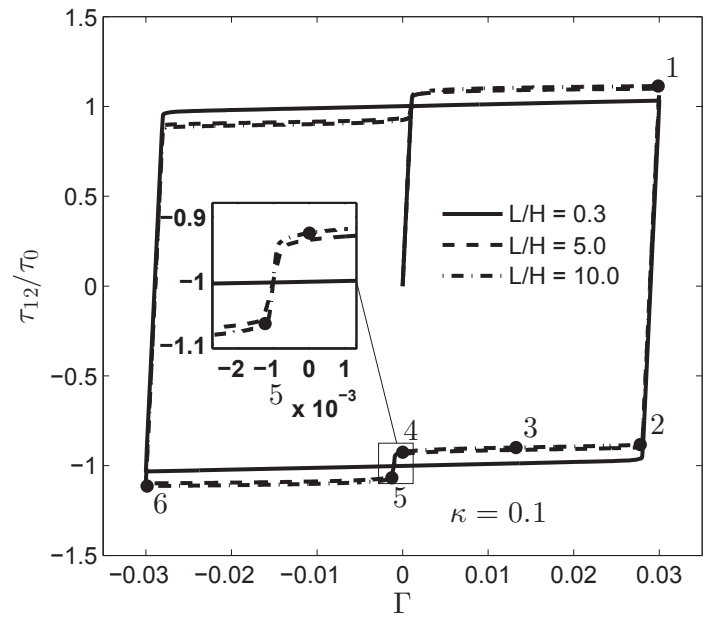

(b)

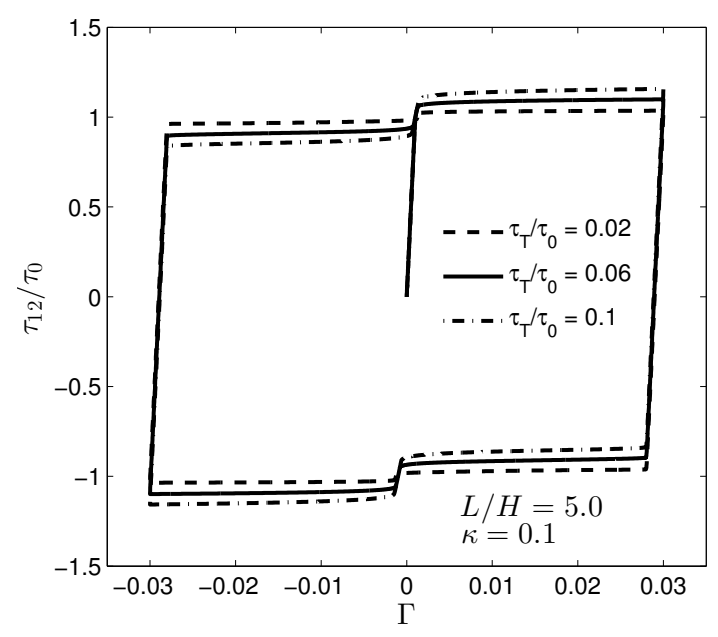

(c)

Figure 5: Numerical results for the single slip simple shear problem (Fig. 1), using the purely phenomenological back stress formulation (Eq. (14)), with $\theta=90^{\circ}, h^{(\alpha \beta)}=h=0, m=0.02$, and $\dot{U}=H \dot{\gamma}_{0}$. The figure shows normalized shear stress vs. macroscopic shear strain: (a) for different values of $\kappa$, (b) for different values of $L$, and (c) for different values of $\tau_{T}$. In (a) and (b), $\tau_{T} / \tau_{0}=0.06$. In (b) the numbered dots mark the load stages at which the slip and back stress are plotted in the subsequent figures. 


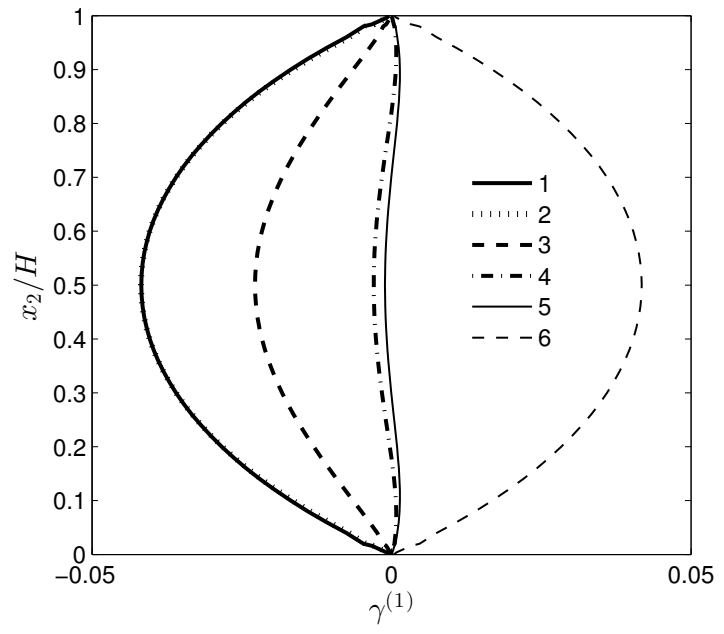

(a)

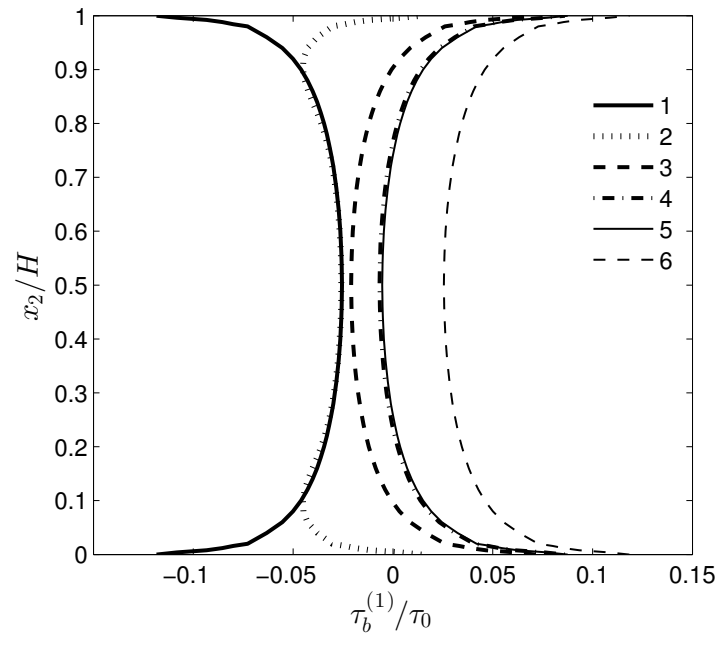

(b)

Figure 6: Numerical results for the single slip simple shear problem (Fig. 1), using the classical quadratic gradient energy based back stress (corresponding to $\mu=\kappa=1$ ), with $\theta=90^{\circ}$, $h^{(\alpha \beta)}=h=0, m=0.02$, and $\dot{U}=H \dot{\gamma}_{0}$, The figure shows (a) slip profiles and (b) back stress distribution at six different stages during cyclic loading corresponding to the points marked on the curve in Fig. 4. 


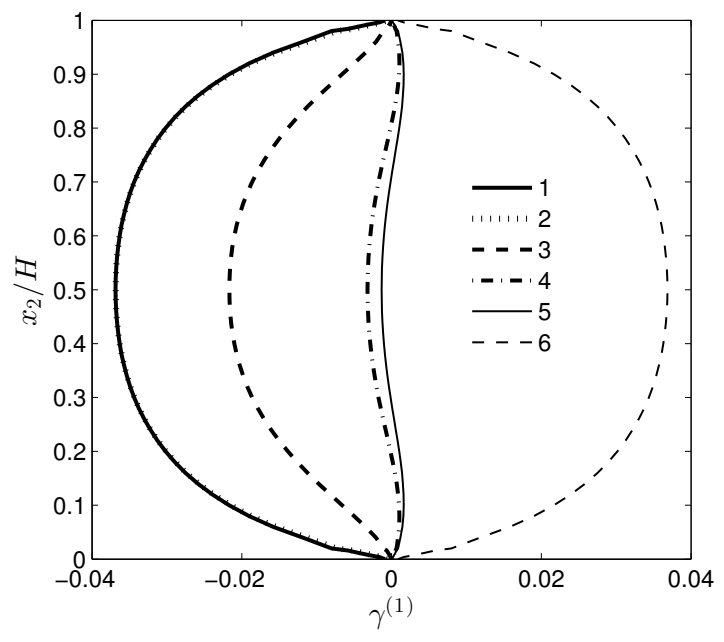

(a)

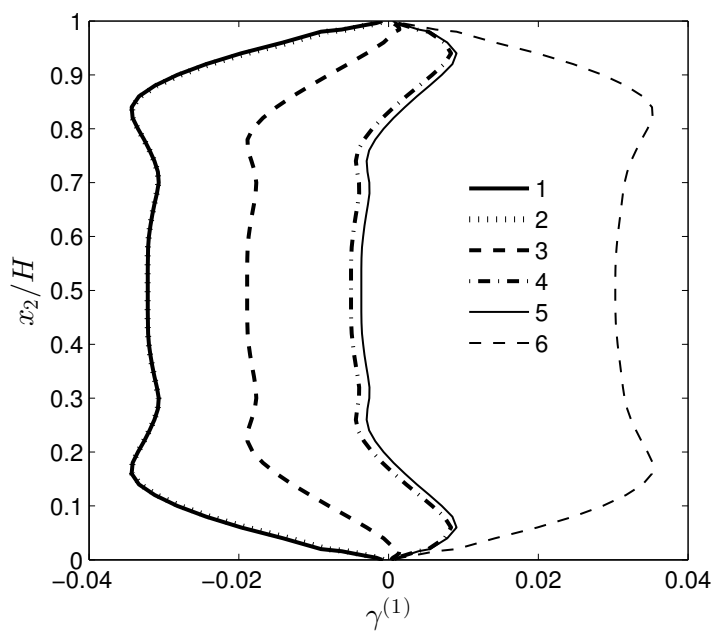

(c)

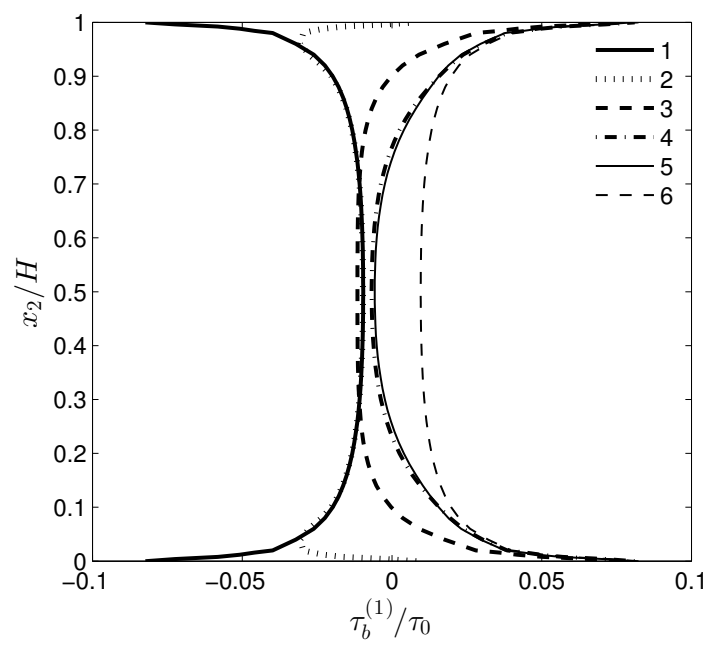

(b)

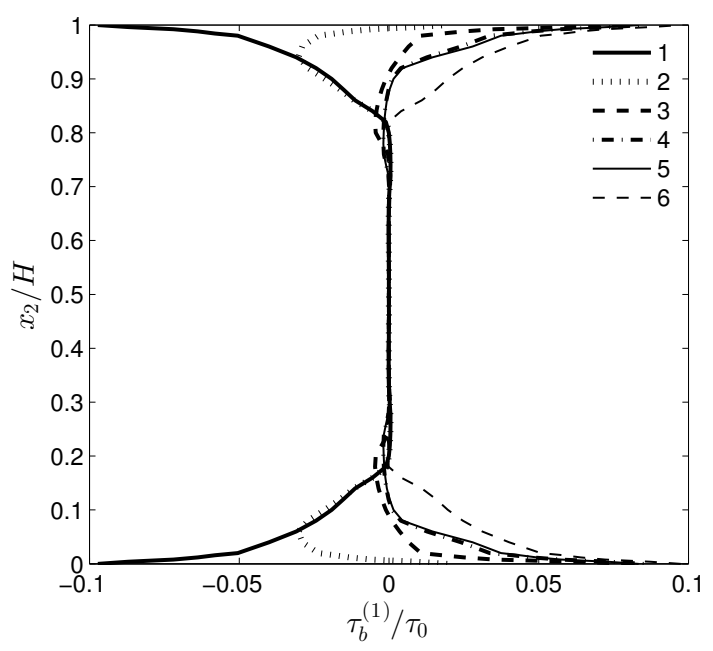

(d)

Figure 7: Numerical results for the single slip simple shear problem (Fig. 1), assuming a nearly linear gradient energy based back stress $(\mu=0.01$ in (a) and (b)) and cubic gradient energy $\left(\mu=2.0\right.$ in (c) and (d)) (Eqs. (12) and (13)), with $\theta=90^{\circ}, h^{(\alpha \beta)}=h=0, m=$ $0.02, L / H=0.3$, and $\dot{U}=H \dot{\gamma}_{0}$. The figure shows slip profiles ((a) and (c)) and back stress distribution ((b) and (d)) at six different stages during cyclic loading corresponding to the points marked on the curve in Fig. 4. 


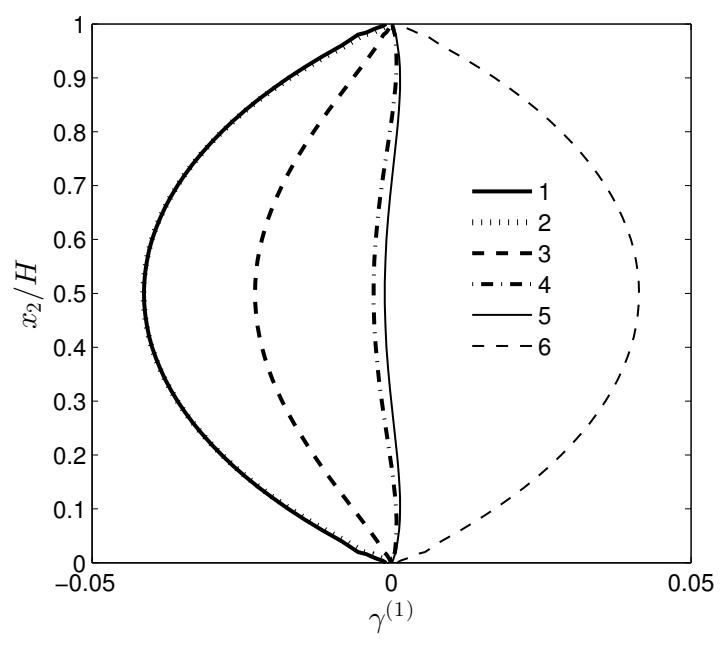

(a)

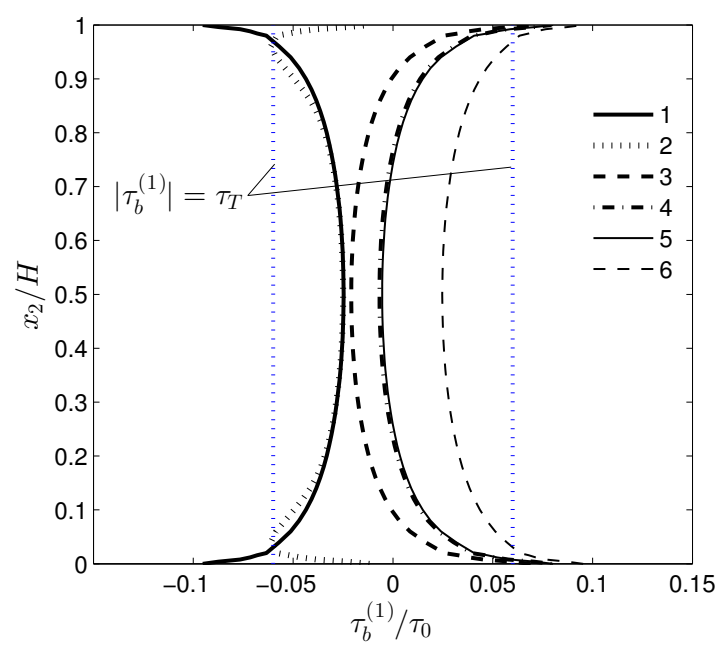

(b)

Figure 8: Numerical results for the single slip simple shear problem (Fig. 1), using the back stress power law (Eq. (14)), with $\theta=90^{\circ}, h^{(\alpha \beta)}=h=0, L / H=0.3, m=0.02, L / H=0.3$, $\kappa=0.1, \tau_{T} / \tau_{0}=0.06$, and $\dot{U}=H \dot{\gamma}_{0}$. The figure shows (a) slip profiles, and (b) back stress distribution at six different stages during cyclic loading corresponding to the points marked on the curve in Fig. 5.

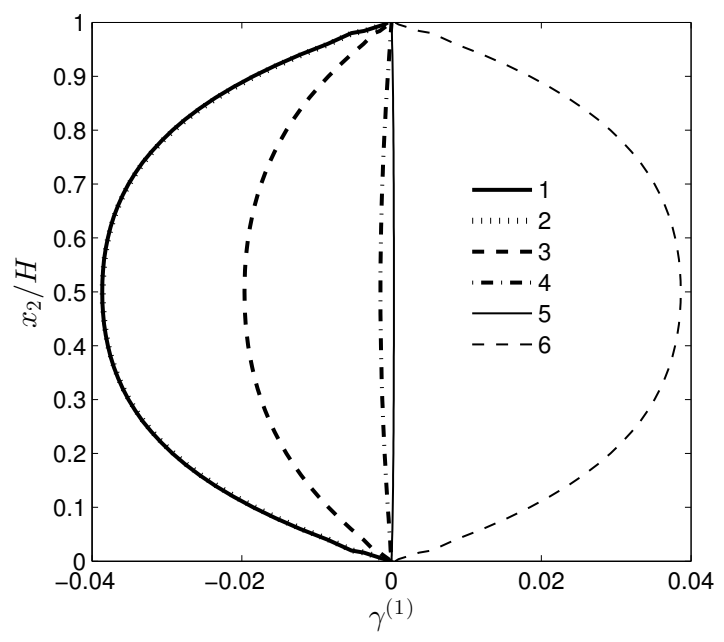

(a)

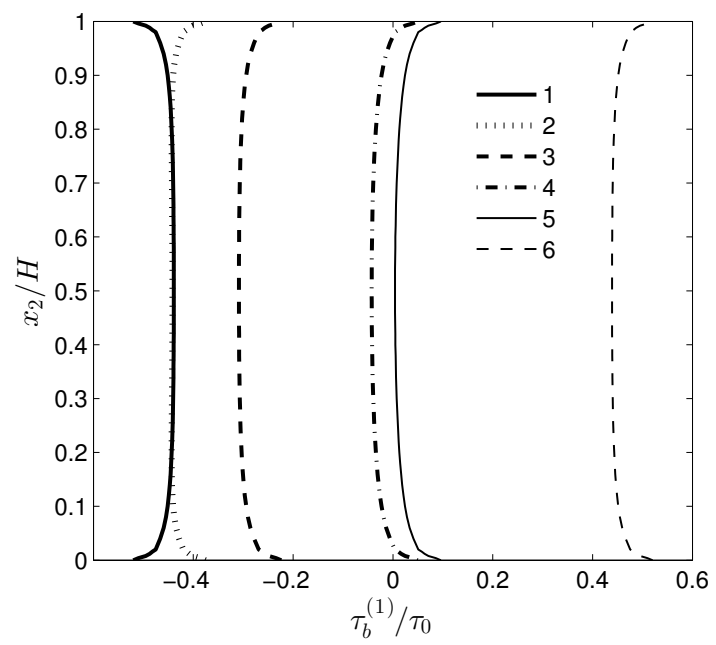

(b)

Figure 9: Numerical results for the single slip simple shear problem (Fig. 1), assuming a nearly linear gradient energy based back stress (Eqs. (12) and (13)), with $\theta=90^{\circ}, h^{(\alpha \beta)}=$ $h=0, m=0.02, L / H=10.0, \mu=0.01$, and $\dot{U}=H \dot{\gamma}_{0}$. The figure shows (a) slip profiles, and (b) back stress distribution at six different stages during cyclic loading corresponding to the points marked on the curve in Fig. 4. 


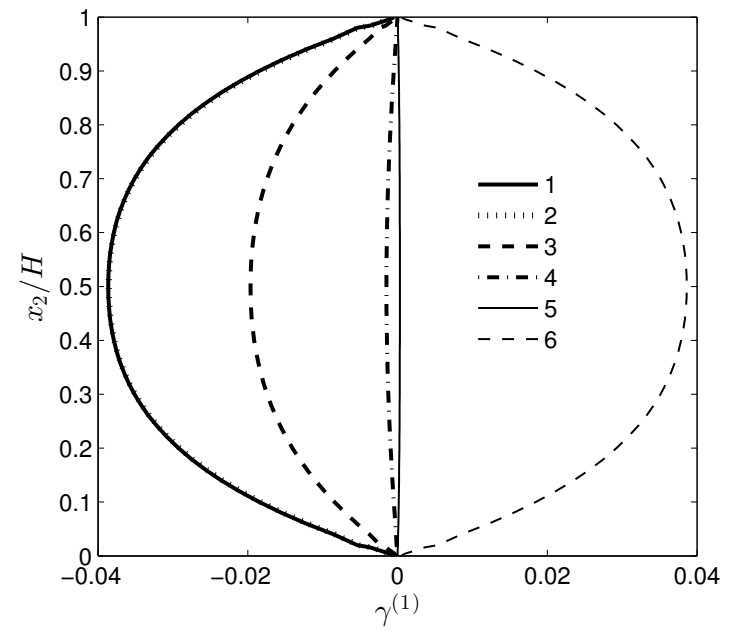

(a)

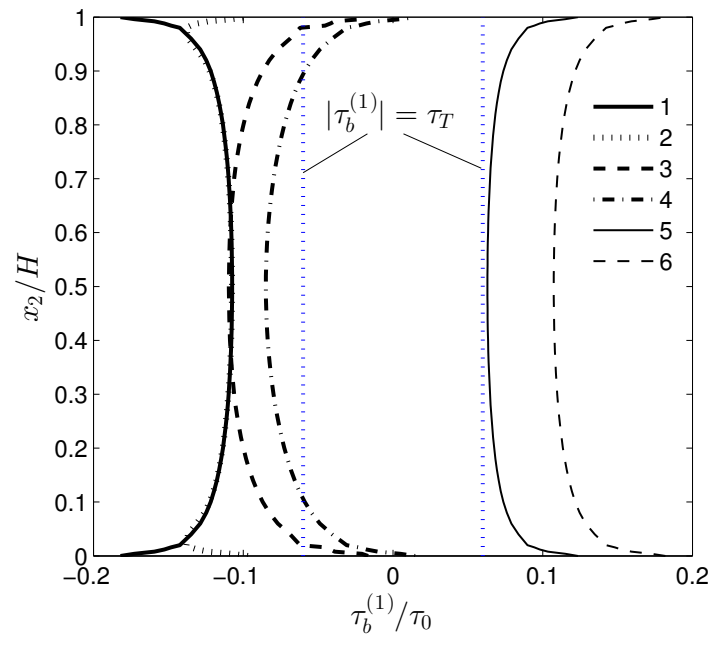

(b)

Figure 10: Numerical results for the single slip simple shear problem (Fig. 1), using the back stress power law (Eq. (14)), with $\theta=90^{\circ}, h^{(\alpha \beta)}=h=0, m=0.02, L / H=10.0, \kappa=$ $0.1, \tau_{T} / \tau_{0}=0.06$, and $\dot{U}=H \dot{\gamma}_{0}$. The figure shows (a) slip profiles, and (b) back stress distribution at six different stages during cyclic loading corresponding to the points marked on the curve in Fig. 5.

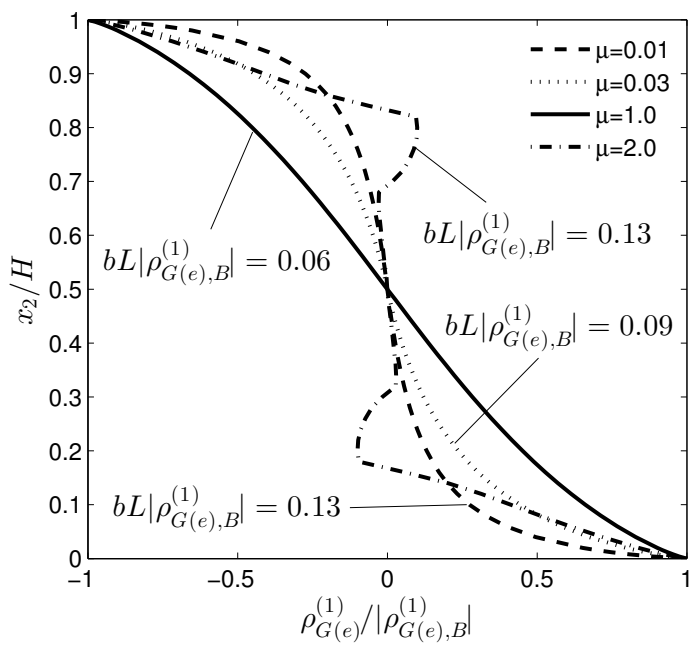

Figure 11: Predicted GND micro-structure, at $\Gamma=0.03$ after two load cycles, assuming a nearly linear (dashed line, $\mu=0.01$ and dotted line, $\mu=0.03$ ), quadratic (solid line, $\mu=$ 1.0), and cubic (dashed-dotted line, $\mu=2.0$ ) gradient energy based back stress (Eqs. (12) and (13)), with $\theta=90^{\circ}, h^{(\alpha \beta)}=h=0, m=0.02, L / H=0.3$, and $\dot{U}=H \dot{\gamma}_{0}$. 


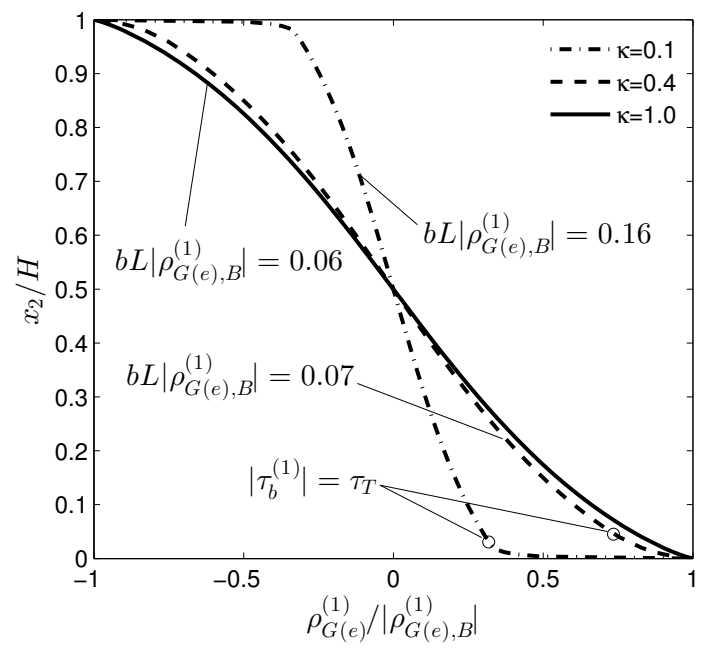

Figure 12: Predicted GND micro-structure, at $\Gamma=0.03$ after two load cycles, using the back stress power law (Eq. (14)) with $\kappa=0.1$ and $\kappa=0.4$, and $\tau_{T} / \tau_{0}=0.06$, compared with the the classical quadratic gradient energy based back stress formulation $(\kappa=1.0)$, with $\theta=90^{\circ}$, $h^{(\alpha \beta)}=h=0, m=0.02, L / H=0.3$, and $\dot{U}=H \dot{\gamma}_{0}$. Circles mark the back stress transition point $\left(\left|\tau_{b}^{(1)}\right|=\tau_{T}\right)$ near the lower horizontal boundary. 